University of Nebraska - Lincoln

DigitalCommons@University of Nebraska - Lincoln

Faculty Publications, Department of Physics and Astronomy

Research Papers in Physics and Astronomy

$7-1-2004$

\title{
OPTICAL VARIABILITY OF NARROW-LINE SEYFERT 1 GALAXIES
}

\author{
Elizabeth S. Klimek \\ University of Nebraska-Lincoln, eklimek@nmsu.edu \\ C. Martin Gaskell \\ University of Nebraska-Lincoln, mgaskell@ucsc.edu \\ Cecilia H. Hedrick \\ University of Nebraska-Lincoln
}

Follow this and additional works at: https://digitalcommons.unl.edu/physicsfacpub

Part of the Physics Commons

Klimek, Elizabeth S.; Gaskell, C. Martin; and Hedrick, Cecilia H., "OPTICAL VARIABILITY OF NARROW-LINE SEYFERT 1 GALAXIES" (2004). Faculty Publications, Department of Physics and Astronomy. 5. https://digitalcommons.unl.edu/physicsfacpub/5

This Article is brought to you for free and open access by the Research Papers in Physics and Astronomy at DigitalCommons@University of Nebraska - Lincoln. It has been accepted for inclusion in Faculty Publications, Department of Physics and Astronomy by an authorized administrator of DigitalCommons@University of Nebraska Lincoln. 


\title{
OPTICAL VARIABILITY OF NARROW-LINE SEYFERT 1 GALAXIES
}

\author{
Elizabeth S. Klimek, C. Martin Gaskell, and Cecelia H. Hedrick \\ Department of Physics and Astronomy, University of Nebraska, Lincoln, NE 68588-0111; \\ penumbra89@hotmail.com,mgaskell1@unl.edu, piqueen314@hotmail.com \\ Received 2003 December 22; accepted 2004 March 3
}

\begin{abstract}
We present results of a broadband photometric study of the optical variability of six narrow-line Seyfert 1 (NLS1) galaxies observed at 172 epochs. We searched for microvariability on 33 nights. Strong evidence for microvariability was found only for our lowest luminosity object, NGC 4051, on one night. Weaker evidence suggests such variability on a few other nights for two other objects, but the data are not as convincing. Intranight variability in NLS1's is thus concluded to be rare and of low amplitude. We give illustrations of how variable image quality can produce spurious variability. We find that for well-studied non-NLS1's there is a spread in the amplitude of seasonal variability (i.e., in some years an active galactic nucleus [AGN] is more variable than in others). We find that the means of the variability amplitudes of non-NLS1's over several seasons vary from object to object (i.e., some AGNs are, on average, more variable than others). NLS1's also show a spread in seasonal variabilities. The best-studied NLS1, Ark 564, shows a range of amplitudes of variability from season to season that is comparable to the range found in broad-line Seyfert 1 galaxies (BLS1's), and in one season Ark 564 was as variable as the most variable non-NLS1. The seasonal amplitudes of variability for NLS1's are mostly in the lower half of the range of non-BLS1 seasonal amplitudes, but the absence of a suitable control sample makes a precise comparison difficult. However, on long timescales (weeks to years) NLS1's as a class are not more variable than non-NLS1's. The extreme variability seen in the X-rays was not seen in the optical. This has consequences for the models of AGNs in general as well as NLS1's in particular.
\end{abstract}

Subject headings: accretion, accretion disks — black hole physics — galaxies: active — quasars: general — X-rays: galaxies

On-line material: machine-readable tables

\section{INTRODUCTION}

It has long been realized that some active galactic nuclei (AGNs) have only narrow emission lines in their spectra while at the same time showing the characteristic spectrum of the broad-line region (BLR; e.g., Phillips 1977; Koski 1978; Davidson \& Kinman 1978). Thus, the BLR (high density) lines were narrower than usual. These objects came to be called narrow-line Seyfert 1 galaxies (NLS1's; Gaskell 1984; Osterbrock \& Pogge 1985; Goodrich 1989). Although there is no sharp demarcation, an AGN is commonly called an NLS1 if the broad lines have an FHWM $\leq 2000 \mathrm{~km} \mathrm{~s}^{-1}$. We refer to AGNs with FWHM $>2000 \mathrm{~km} \mathrm{~s}^{-1}$ as BLS1's ("broad-line Seyfert 1 galaxies"). The properties of NLS1's lie at one end of a set of correlations between AGN properties that is commonly called eigenvector 1 (Boroson \& Green 1992). In addition to the curiously narrow "broad" lines, NLS1's tend to have steeper soft X-ray spectra than non-NLS1's (Boller et al. 1996). Many exhibit rapid soft X-ray variability, which can also be large in amplitude, such as a factor of $\sim 100$ in a day in IRAS 13224-3809 (Boller et al. 1997). NLS1's show strong optical Fe II emission lines (Sargent 1968) and sometimes the higher ionization Fe lines (Davidson \& Kinman 1978), but while the Fe II equivalent widths are about the same as those of non-NLS1's (Gaskell 1985), the $\mathrm{H} \beta$ equivalent widths are smaller than those of non-NLS1's (Gaskell 1985; Goodrich 1989). The $[\mathrm{O}$ III $] / \mathrm{H} \beta$ line ratio is less than 3 , which is less than the dividing line that Shuder \& Osterbrock (1981) found between Seyfert 1 and 2 galaxies (i.e., the $[\mathrm{O}$ III] $/ \mathrm{H} \beta$ ratio implies that they are Seyfert 1 galaxies).
A common trait possessed by all AGNs is that they display some degree of variability (Ulrich et al. 1997; Gaskell \& Klimek 2003). The most vigorous variability observed is that of the X-rays (Mushotsky et al. 1993). Variability is valuable in that it provides clues to understanding what AGNs actually are and how they work. Details of the inner workings of AGNs remain poorly understood, despite over three decades of research. Variability can help set constraints on the sizes of different regions of AGNs and can give information about the processes that are driving the variations. There has been much effort in looking for optical variability, some of it in conjunction with monitoring in other wavebands. If a link is found between the variability in different wavebands, then the processes behind each kind of variability are probably related. Explanations of AGN variability include hot spots on accretion disks, flares, and relativistic jets. BL Lac objects and optically violently variable AGNs (OVVs) exhibit strong variability on short timescales. This is believed to be a consequence of relativistic beaming by jets. The beaming can amplify intrinsic variations, which may or may not originate from within the jet.

Rapid (subdiurnal) variability in the X-ray emission of AGNs is well known, but there have been conflicting claims about the frequency of occurrence of rapid intranight optical variability. Thus far, observing campaigns searching for optical variability in AGNs have typically yielded low-amplitude variations on timescales of no less than a few days (e.g., Webb \& Malkan 2000a). De Ruiter \& Lub (1986) did not find any rapid $(<1$ day) variations of greater than $0.5 \%$ for any of the eight Seyfert galaxies they observed, while other observers 
TABLE 1

Comparison Stars

\begin{tabular}{|c|c|c|c|}
\hline Object & Star & $\begin{array}{c}V \\
(\mathrm{mag})\end{array}$ & Reference \\
\hline \multirow[t]{4}{*}{ Ark $564 \ldots \ldots \ldots \ldots \ldots . . .}$. & 0 & 12.175 & \multirow[t]{4}{*}{1} \\
\hline & 1 & 13.658 & \\
\hline & 3 & 14.176 & \\
\hline & 5 & 14.490 & \\
\hline \multirow[t]{2}{*}{ Mrk $478 \ldots \ldots \ldots \ldots \ldots$} & 2 & 14.255 & \multirow[t]{2}{*}{ Uncertain to \pm 0.2} \\
\hline & 3 & 13.495 & \\
\hline \multirow[t]{4}{*}{ Mrk 493 ................. } & 2 & 13.107 & \multirow{4}{*}{ Uncertain to \pm 0.2} \\
\hline & 4 & 13.684 & \\
\hline & 5 & 13.933 & \\
\hline & 8 & 14.429 & \\
\hline \multirow[t]{2}{*}{ Mrk 335 ................ } & 4 & 13.679 & \multirow[t]{2}{*}{2} \\
\hline & 6 & 13.794 & \\
\hline \multirow[t]{4}{*}{ Mrk $359 \ldots \ldots \ldots \ldots . . . .}$. & 1 & 13.473 & \multirow[t]{4}{*}{ Uncertain to \pm 0.2} \\
\hline & 3 & 13.533 & \\
\hline & 4 & 13.725 & \\
\hline & 5 & 14.540 & \\
\hline \multirow[t]{4}{*}{ NGC $4051 \ldots \ldots \ldots \ldots . . .}$. & 1 & 14.010 & \multirow[t]{3}{*}{3} \\
\hline & 2 & 11.120 & \\
\hline & 4 & 13.390 & \\
\hline & 5 & 14.760 & Our calibration (not used) \\
\hline
\end{tabular}

REFERENCES.-(1) C. M. Gaskell et al. 2004, in preparation; (2) Shrader et al. 1990; (3) Penston et al. 1971.

have reported low-level microvariability on subdiurnal timescales. For example, Merkulova (2000) reported subdiurnal variability at the $1 \%$ level on $60 \%$ of nights for NGC 4151 . Jang \& Miller $(1995,1997)$ found that eight of 17 radio-quiet and six of seven radio-loud AGNs showed intranight variability on the order of a few percent (see also Carini et al. 2003). Merkulova (2000) concluded that intranight variability is transient in character and has manifested itself with different probabilities for different galaxies. Even though there is some uncertainty over the frequency of occurrence of subdiurnal variability, it would seem that extremely rapid and/or largeamplitude optical variations are rare.

The most extreme X-ray variability in non-OVV AGNs is seen in NLS1's. The shortest timescales for X-ray variability are about 200-1000 s (Boller et al. 1993). Over a short period of time, the amplitude can change by as much as a factor of 4 , as seen in the NLS1 IRAS 13224-3809, which varied by this much over a course of hours (Boller et al. 1997). Largeamplitude rapid X-ray variability is one of the interesting properties of NLS1's. It has been suggested that NLS1's are beamed (Boller et al. 1997), since they display similarly strong variations in the X-rays.

If rapid optical variations are present in AGNs other than BL Lacs, it is possible that they would most likely be found in objects displaying the most extreme X-ray variability, namely, the NLS1's. On long timescales Giannuzzo \& Stirpe (1996) and Giannuzzo et al. (1998) compared the Balmer line variability of NLS1's with that of NGC 5548 and found the NLS1's to be less variable. However, this was not a general comparison with non-NLS1's, and it was possible that NGC 5548 was more variable than the average BLS1. Young et al. (1999) unsuccessfully searched for intranight optical variability in IRAS 13224-3809, while Miller et al. (2000) reported dramatic variability in the same object on one night.

The work presented here has two goals: (1) to look for evidence of subdiurnal optical continuum variations, especially those of large amplitude, in a larger sample of NLS1's

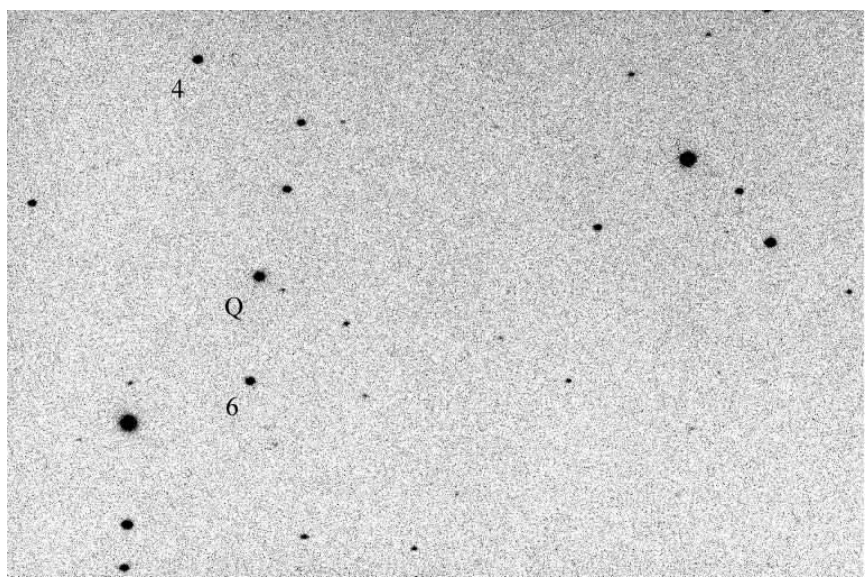

FIG. 1.-Comparison stars for Mrk 335.

and (2) to see whether NLS1's are more variable in the optical than non-NLS1's on longer timescales. For one of the objects in our sample, Ark 564, there are observations over a much longer period of time, so we discuss this object at more length in a separate paper (C. M. Gaskell et al. 2004, in preparation).

\section{OBSERVATIONS}

The majority of the observations were made with the University of Nebraska's $0.4 \mathrm{~m}$ Schmidt-Cassegrain telescope at the university's Lincoln Observatory in a bright sky location. Images were recorded with a Kodak KAF-0401 CCD, giving 0 ".887 pixel $^{-1}$ through an $\mathrm{f} / 5$ focal reducer. All images were taken through a standard Johnson $V$ filter. Additional observations were made with the $0.8 \mathrm{~m}$ Cassegrain telescope at the university's Behlen Observatory near Mead, Nebraska, in a darker sky location, with a Kodak KAF-1001E chip giving 0 ".59 pixel $^{-1}$ with an $\mathrm{f} / 9$ focal reducer through an identical filter. The NLS1's and the comparison stars were measured through photometric apertures of identical effective solid angles, and we found that no scaling was necessary between observations from the two telescopes.

A sample of six NLS1's was chosen only on the basis of the criteria that they had to be bright enough to be observed with the $0.4 \mathrm{~m}$ telescope and that they had to be high-declination sources in order to maximize the amount of time that they would be accessible during the night. Thus, our selection was not based on any other specific property or characteristic of this class of AGNs. In particular, variability history was not taken into consideration. The objects selected were Ark 564, Mrk 478, Mrk 493, Mrk 335, Mrk 359, and NGC 4051.

Observations for most objects were taken between 2000 May and 2003 June, with the exception of Ark 564, for which the observations considered here began in 1998 August. In searching for intranight variations, we observed objects continuously for most of a night. Integration times were 1015 minutes on the $0.4 \mathrm{~m}$ telescope and 5 minutes on the $0.8 \mathrm{~m}$ telescope. Anywhere from 3 to 26 images per object were obtained during any one night, giving a range of 30 minutes to $6.5 \mathrm{hr}$ of continuous data for the most intensively observed objects.

\section{DATA REDUCTION}

\subsection{Comparison Stars}

A summary of the adopted magnitudes for the comparison stars used for the NLS1's (except Ark 564; see C. M. Gaskell 


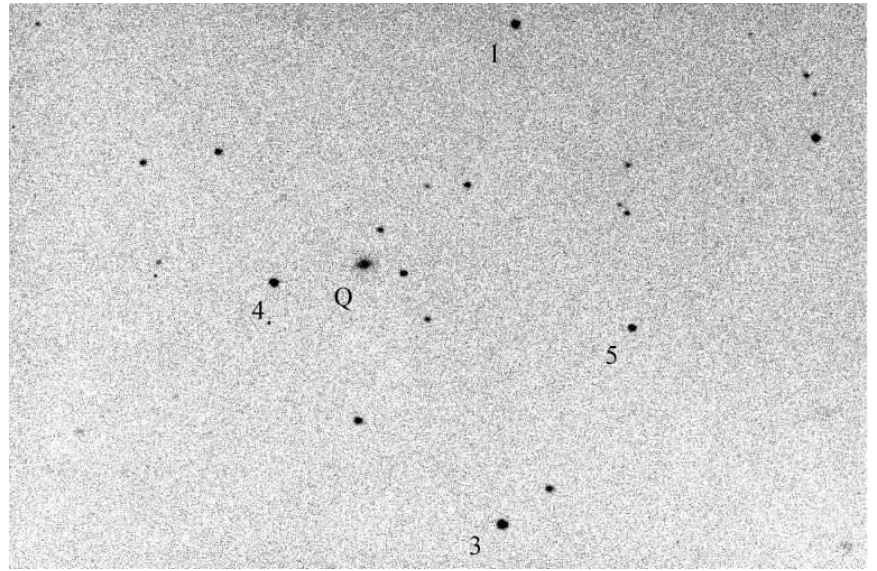

FIG. 2.-Comparison stars for Mrk 359.

et al. 2004, in preparation) is given in Table 1, and finding charts are shown in Figures 1-5. Where possible, comparison stars were chosen from the literature. For Mrk 359, Mrk 493, and Mrk 478 we calibrated our own comparison stars from the count rates on a handful of our best nights. The relative calibration of these stars is good, but the absolute calibration is believed to be uncertain by $\pm 0.2 \mathrm{mag}$. This uncertainty has no impact on this study, as we are interested only in changes in magnitudes. In the case of Mrk 478, our magnitudes agree with the relative magnitudes given by Webb \& Malkan (2000b).

The detector noise is non-Gaussian because of hot pixels and cosmic rays. If one comparison star gave anomalous magnitudes for an image, it was not used for that image. Likewise, if the average magnitude for an AGN was significantly off for an image on a night, it was dropped from the nightly average. Between $5 \%$ and $10 \%$ of comparisons were discarded. Visual inspection often revealed obvious image problems in these cases.

\subsection{Size of Photometric Aperture}

In order to maximize the accuracy with which the counts from an object are measured with respect to a sky background, the size of the measuring aperture should be small in order to reduce the sky background contribution but, at the same time, large enough to get a good signal-to-noise ratio and to minimize the effect of fluctuations in the measurement due to

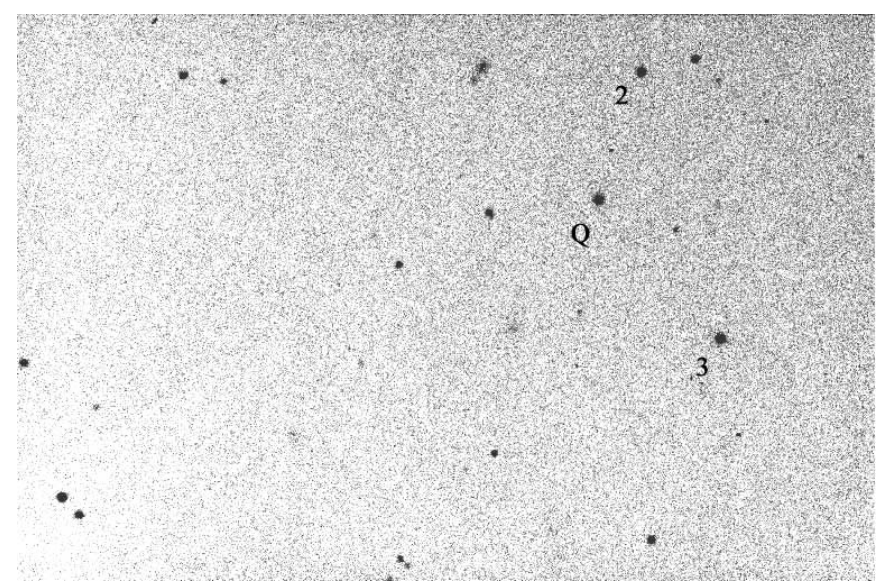

FIG. 3.-Comparison stars for Mrk 478.

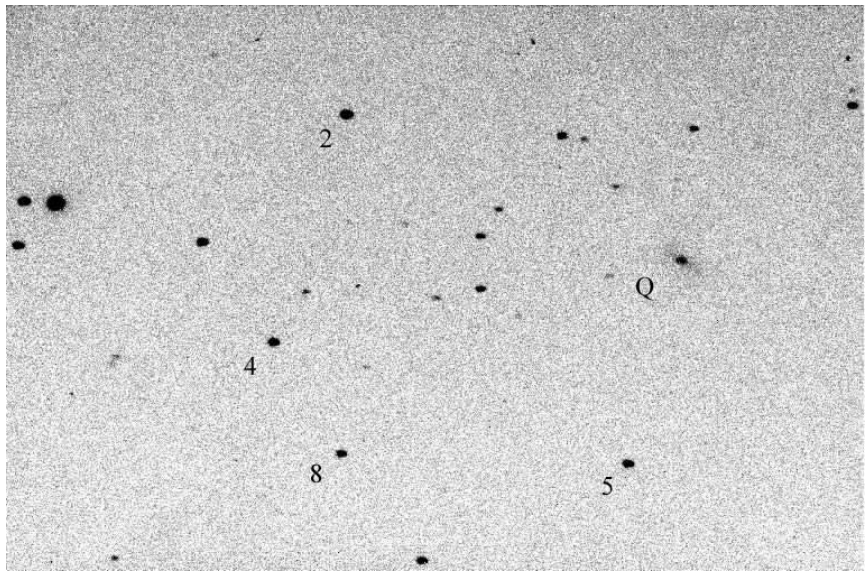

FIG. 4.-Comparison stars for Mrk 493.

miscentering. A compromise must then be chosen in order to obtain the best measurement.

For point sources in stellar photometry, these effects have been studied by Howell (1989). For faint stellar sources, the optimum aperture radius is about 3 pixels or about the FWHM. A number of previous studies of AGN variability have followed the Howell prescription (e.g., Carini et al. 2003; Jang \& Miller 1995, 1997), but, as Cellone et al. (2000) pointed out, the effects of the underlying host galaxy, especially in lowluminosity AGNs, should not be ignored. Because of the underlying host galaxy, even small seeing fluctuations can introduce spurious variability in the AGN flux that can be mistaken for microvariability. This is because poor seeing turns a point image into a extended image, but a galaxy already has an extended image and is therefore affected much less. This means that poor image quality causes more light loss from a circular aperture for a star than for a galaxy, and thus the galaxy will appear to be brighter relative to the star. We provide illustrations of this effect below. Similar conclusions in the spectroscopic case of choosing a suitable aperture were found by Peterson et al. (1995).

Since the ideal aperture is a function of the radial brightness distribution of the galaxy and its brightness relative to the AGN, we experimented with two aperture sizes to find the one most appropriate for a given object. The larger aperture had a radius of 9 pixels on the $0.4 \mathrm{~m}$ telescope, corresponding to $8^{\prime \prime}$, and the smaller aperture had a radius of 5 pixels, or 4 ". 4 . The

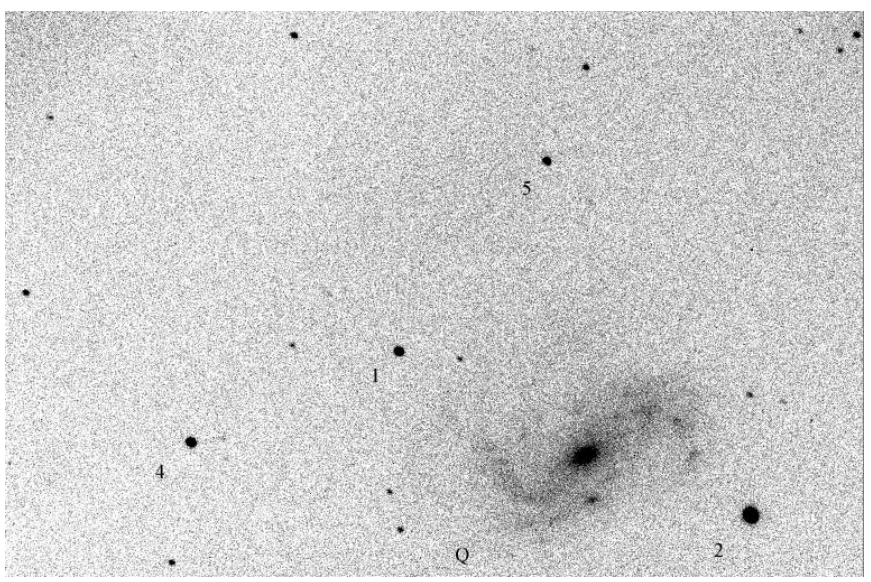

FIG. 5.-Comparison stars for NGC 4051 
TABLE 2

Mrk $335 V$ Magnitudes during Nights Searched for Microvariability

\begin{tabular}{ccccc}
\hline \hline UT & JD & $\begin{array}{c}V \\
(\mathrm{mag})\end{array}$ & $\begin{array}{c}\text { Error } \\
( \pm)\end{array}$ & $\begin{array}{c}\text { FWHM } \\
(\operatorname{arcsec})\end{array}$ \\
\hline 2002 Oct $13 \ldots \ldots \ldots . .$. & $2,452,560.73$ & 13.585 & 0.013 & 4.3 \\
& $2,452,560.74$ & 13.605 & 0.013 & 4.6 \\
& $2,452,560.75$ & 13.587 & 0.013 & 4.7 \\
& $2,452,560.76$ & 13.583 & 0.013 & 4.7 \\
& $2,452,560.77$ & 13.568 & 0.013 & 5.1
\end{tabular}

NoтE.-Table 2 is published in its entirety in the electronic edition of the Astrophysical Journal. A portion is shown here for guidance regarding its form and content.

same sky annulus was kept in both cases, originally chosen to be appropriate for the larger aperture size, in order to exclude most of the galaxy component from the sky background measurement. The annulus had an inner radius of 13.5 pixels $\left(12^{\prime \prime}\right)$ and an outer radius of up to 22.5 pixels $\left(20^{\prime \prime}\right)$.

Each object was measured with both apertures, and the resulting errors were analyzed. Here we give only the measurements resulting from the aperture that produced the lowest rms variation in the errors of the nightly means for each object. For all objects but Mrk 478 (one of our faintest and most compact objects) the larger aperture of $8^{\prime \prime}$ was used. A summary of our individual observations on nights we searched for microvariability is presented in Tables 2-6. We discuss the estimation of our errors below. For other nights just the nightly means are given in Table 7 .

\section{ANALYSIS}

There are several sources that can give rise to spurious variability. These sources include imperfect flat-fielding, possible inconstancy of comparison stars, and changes in image quality due to seeing and focus shift. The latter causes fluctuation in the galaxy component measured (see above).

\subsection{Estimation of Errors}

Photon statistics alone underestimates errors, so we used other methods to estimate the errors. For the long-term light curves, the error for each night was calculated by dividing the standard deviation $(\sigma)$ of the magnitudes by the square root of the number of images $(n)$ for the night. Nights with few images typically have the greater error, but sometimes such nights had fortuitously small errors (e.g., if two or three of the magnitudes happened to be essentially the same). In these cases the individual night's $\sigma$ was replaced by the median standard deviation $\sigma_{\text {med }}$ for all of an object's nights of

TABLE 3

Mrk 359 V Magnitudes During Nights Searched for Microvariability

\begin{tabular}{ccccc}
\hline \hline UT & JD & $\begin{array}{c}V \\
(\mathrm{mag})\end{array}$ & $\begin{array}{c}\text { Error } \\
( \pm)\end{array}$ & $\begin{array}{c}\text { FWHM } \\
(\operatorname{arcsec})\end{array}$ \\
\hline 2002 Nov 07 …….. & $2,452,585.75$ & 13.900 & 0.023 & 4.9 \\
& $2,452,585.76$ & 13.910 & 0.023 & 4.6 \\
& $2,452,585.77$ & 13.854 & 0.023 & 4.3 \\
& $2,452,585.78$ & 13.885 & 0.023 & 4.3 \\
& $2,452,585.80$ & 13.886 & 0.023 & 3.5 \\
\hline
\end{tabular}

NoтE.-Table 3 is published in its entirety in the electronic edition of the Astrophysical Journal. A portion is shown here for guidance regarding its form and content.
TABLE 4

Mrk $478 V$ Magnitudes during Nights Searched for Microvariability

\begin{tabular}{ccccc}
\hline \hline UT & JD & $\begin{array}{c}\mathrm{V} \\
(\mathrm{mag})\end{array}$ & $\begin{array}{c}\text { Error } \\
( \pm)\end{array}$ & $\begin{array}{c}\text { FWHM } \\
(\operatorname{arcsec})\end{array}$ \\
\hline 2002 Mar 12........ & $2,452,345.76$ & 16.179 & 0.017 & 3.7 \\
& $2,452,345.77$ & 16.148 & 0.017 & 3.6 \\
& $2,452,345.78$ & 16.162 & 0.017 & 3.4 \\
& $2,452,345.79$ & 16.145 & 0.017 & 3.3 \\
& $2,452,345.80$ & 16.174 & 0.017 & 3.3
\end{tabular}

NotE.-Table 4 is published in its entirety in the electronic edition of the Astrophysical Journal. A portion is shown here for guidance regarding its form and content.

observation. The $\sigma_{\text {med }}$ was thus used as a more likely estimator for calculating each of these nights' errors in order to prevent a serious underestimation of the errors.

This method of estimating errors obviously cannot be used when one is searching for intranight variability, since it calculates the errors under the assumption of no variability. Looking at the differential light curves of stars comparable in brightness to the AGN is a useful and important check but gives only a lower limit to the error because the additional error due to the host galaxy (see above) is not taken into consideration.

In order to estimate the errors on nights that were searched for intranight variability, the following method was used. The difference of adjacent magnitudes in the time series, $\Delta m_{i}=$ $m_{i}-m_{i-1}$, was used to find the point-to-point variation during the night. The standard deviation of this difference was then divided by $\sqrt{2}$, since a difference of two magnitudes was taken, to give the estimated error.

If there is no variability during the course of the night, then this error will be indistinguishable from the nightly standard deviation. This was in fact the case for all of our intranight variability search nights, with the exception of the one night on which intranight variations were detected for NGC 4051.

The difference method could give erroneously large errors if any rapid variations are present on a microvariable timescale of 10-15 minutes. However, we consider this to be unlikely (see below).

\subsection{Searching For Variability}

While it is difficult to rule out microvariability, it is easier to detect or rule out variability on longer timescales (hours). AGNs are already known to vary on timescales of more than 1 day. Therefore, it can be reasoned that if AGNs show variability on a smaller timescale of minutes, then they will show variability on the intermediate timescale of hours.

TABLE 5

Mrk $493 V$ Magnitudes during Nights Searched for Microvariability

\begin{tabular}{ccccc}
\hline \hline UT & JD & $\begin{array}{c}V \\
(\mathrm{mag})\end{array}$ & $\begin{array}{c}\text { Error } \\
( \pm)\end{array}$ & $\begin{array}{c}\text { FWHM } \\
(\operatorname{arcsec})\end{array}$ \\
\hline 2002 Jun 27 .......... & $2,452,452.74$ & 14.977 & 0.058 & 4.0 \\
& $2,452,452.75$ & 14.973 & 0.058 & 3.8 \\
& $2,452,452.77$ & 14.901 & 0.058 & 3.8 \\
& $2,452,452.78$ & 14.839 & 0.058 & 4.1 \\
& $2,452,452.79$ & 14.915 & 0.058 & 4.6
\end{tabular}

NotE.-Table 5 is published in its entirety in the electronic edition of the Astrophysical Journal. A portion is shown here for guidance regarding its form and content. 
TABLE 6

NGC $4051 V$ Magnitudes during Nights Searched for Microvariability

\begin{tabular}{ccccc}
\hline \hline UT & JD & $\begin{array}{c}V \\
(\mathrm{mag})\end{array}$ & $\begin{array}{c}\text { Error } \\
( \pm)\end{array}$ & $\begin{array}{c}\text { FWHM } \\
(\operatorname{arcsec})\end{array}$ \\
\hline 2003 Feb $13 \ldots \ldots \ldots .$. & $2,452,683.72$ & 13.355 & 0.010 & 4.9 \\
& $2,452,683.73$ & 13.356 & 0.010 & 4.8 \\
& $2,452,683.75$ & 13.353 & 0.010 & 3.9 \\
& $2,452,683.76$ & 13.340 & 0.010 & 4.4 \\
& $2,452,683.77$ & 13.348 & 0.010 & 4.3 \\
\hline
\end{tabular}

Note.-Table 6 is published in its entirety in the electronic edition of the Astrophysical Journal. A portion is shown here for guidance regarding its form and content.

To search for variability on the timescale of a few hours, two statistical tests were performed. First, the magnitudes for a night were divided into first and second halves. A Student $t$-test was used to evaluate the significance of differences in the mean magnitude between the two equal halves of the night. The second test was to evaluate the significance of the correlation coefficient for the magnitudes versus time. A significant correlation would mean a significant possibility that variations occurred during a night. If either test gave a two-tailed significance of greater than $90 \%$ (i.e., a less than $10 \%$ probability of arising by chance), further investigation was conducted.

The $t$-test and the correlation test are sensitive only to a trend during the night. Other types of variation would be missed (e.g., a rise and fall or sinusoidal oscillations). To search for general variations on a timescale of 30-45 minutes, an $F$-test was conducted to compare the variance of the magnitudes during a night with the variance in their point-to-point differences. This tells whether or not there is significant correlated variability between exposures, which corresponds to 10-15 minute intervals. Again, we conducted further investigation if the $F$-test gave a significance of greater than $90 \%$.

\subsection{Systematic Errors}

In the cases where the above-mentioned statistical tests showed a high level of significance, we investigated the effects of possible sources of systematic error.

\subsubsection{Image-Quality Effects}

As is well known, good flat-fielding is difficult to attain, even when using sky flats. Variations of $1 \%$ or more across the CCD chip were not uncommon. However, this effect was minimized by having several (typically four) comparison stars, by trying to choose comparison stars that were close to the AGN, and by positioning the AGN on the same part of the chip every night as well as possible. Images that were found to

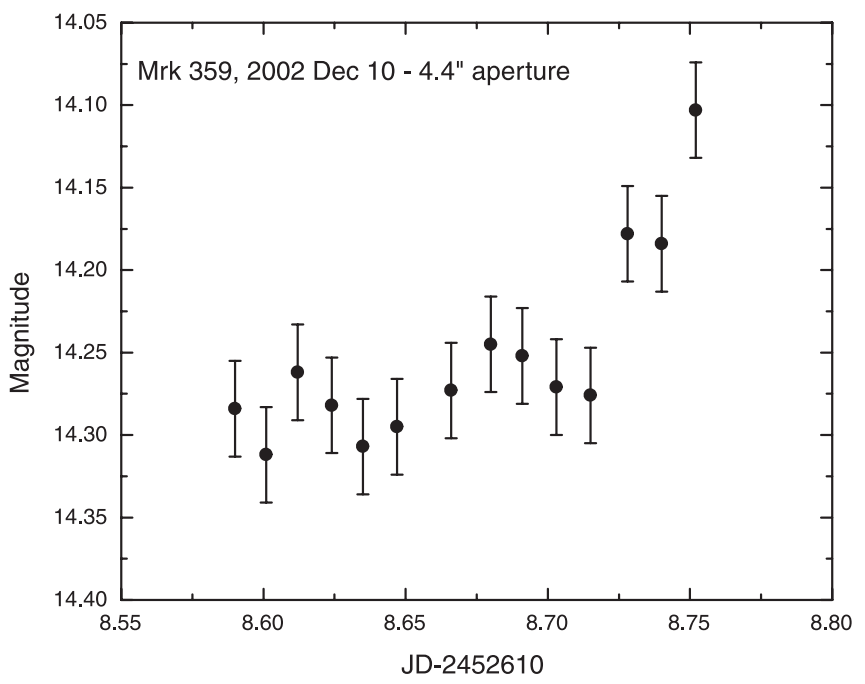

FIG. 6.-Apparent microvariability of Mrk 359 when measured with a 4".4 aperture on 2002 December 10.

have significant flat-fielding problems were removed from our analysis. This sometimes involved dropping entire nights in order to prevent false detections of variations. Where there were signs of possible microvariations, possible flat-fielding problems were investigated by looking at the $(x, y)$-position of the AGN on the chip in addition to visual inspection. If the object "wandered" because of inconsistent positioning during a night, its position was plotted versus time to see if its behavior correlated at all with the object's light curve.

The effect of changes in the image quality due to seeing or focus changes (see $\S 3$ ) was examined by comparing the trend of the average FWHM of the comparison stars during the night with the object's light curve over the same period of time. For each night the AGN magnitudes were also plotted against seeing to further check for any correlation. Such a correlation would signify that any variations present were spurious. Two examples of this analysis are given below.

Only two of the objects, Mrk 359 and Mrk 478, showed a clear FWHM dependence of the magnitudes. This dependence was present only in the 4".4 aperture. No effect was seen in the $8^{\prime \prime}$ aperture. Figures 6-10 show two examples of the seeing dependence during a night of Mrk 359 and Mrk 478 observations. Figures 6 and 9 are plots of the light curves obtained by using a 4".4 aperture. Figure 7 shows the average FWHM of the comparison stars from image to image for Mrk 359 . Figures 8 and 10 confirm the correlations between the magnitudes of the AGNs and the FWHMs. It can be seen that if the changes in image quality were not taken into account,

TABLE 7

Long-Term Light Curves

\begin{tabular}{ccccccc}
\hline \hline Object & UT & JD & Number of Images & $\begin{array}{c}V \\
(\mathrm{mag})\end{array}$ & $\begin{array}{c}\text { Error } \\
( \pm)\end{array}$ & $\begin{array}{r}\text { FWHM } \\
(\operatorname{arcsec})\end{array}$ \\
\hline Mrk 335 .............. & 2001 Oct 02 & $2,452,184.68$ & 3 & 13.409 & 0.012 & 4.6 \\
& 2001 Oct 06 & $2,452,188.74$ & 4 & 13.430 & 0.010 & 2.8 \\
& 2001 Oct 16 & $2,452,198.64$ & 3 & 13.400 & 0.017 & 5.5 \\
& 2001 Oct 20 & $2,452,202.67$ & 4 & 13.495 & 0.010 & 4.0 \\
& 2001 Oct 27 & $2,452,209.58$ & 4 & 13.402 & 0.013 & 3.5 \\
\hline
\end{tabular}

NoтE.-Table 7 is published in its entirety in the electronic edition of the Astrophysical Journal. A portion is shown here for guidance regarding its form and content. 


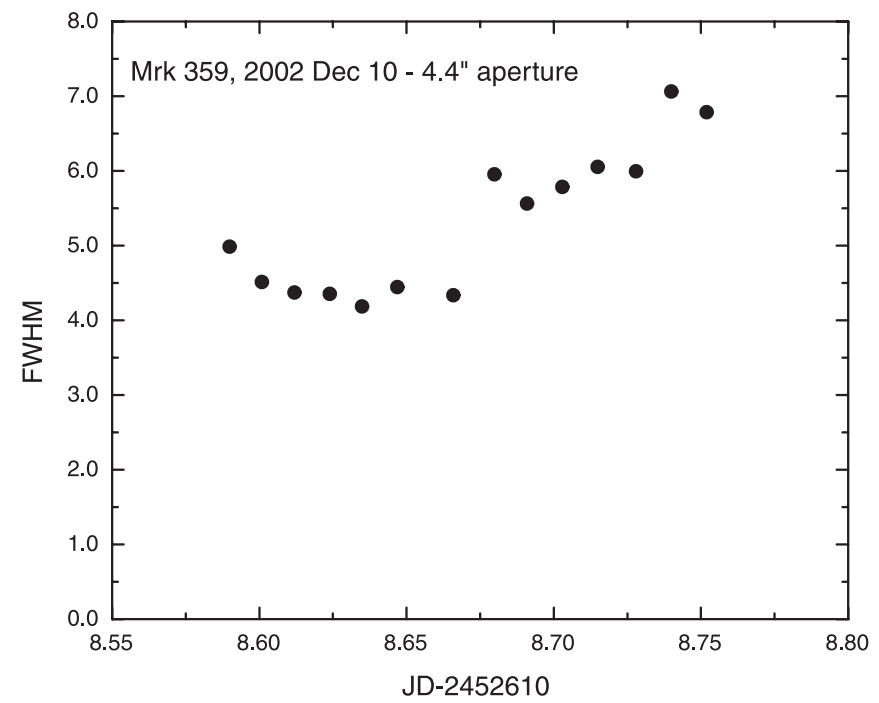

FIG. 7.-Variation of image quality for Mrk 359 on 2002 December 10.

there would be a spurious detection of variability. The statistical tests showed no significant variation for the large apertures, while for Mrk 359, for example, the Student $t$-test was highly significant for the smaller aperture (98\% significance).

Analysis of the errors for all the nights of observations for Mrk 359 showed the least subdiurnal variability for the larger aperture, and this gave the smoothest long-term light curve. However, in the case of Mrk 478, the smaller aperture gave the most consistent errors between nights.

\subsubsection{Constancy of Comparison Stars}

The constancy of the comparison stars was checked by plotting the magnitudes of each individual star with respect to the averages of the other comparison stars for each night. For NGC 4051 star 5 showed evidence of a slow change of $0.03 \mathrm{mag}$ over three observing seasons and was therefore not used. For NGC 4051, for which the only instance of intranight variability was found (see below), it was especially important to have dependably constant stars. There were also slight

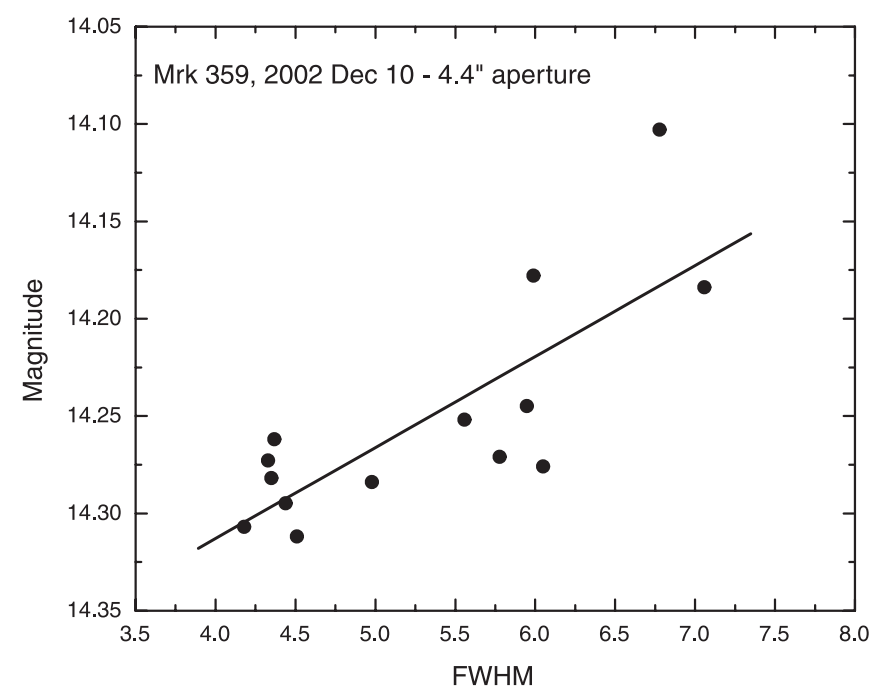

FIG. 8. - Variation of measured magnitude with image quality for Mrk 359 when measured with a 4.4 aperture.

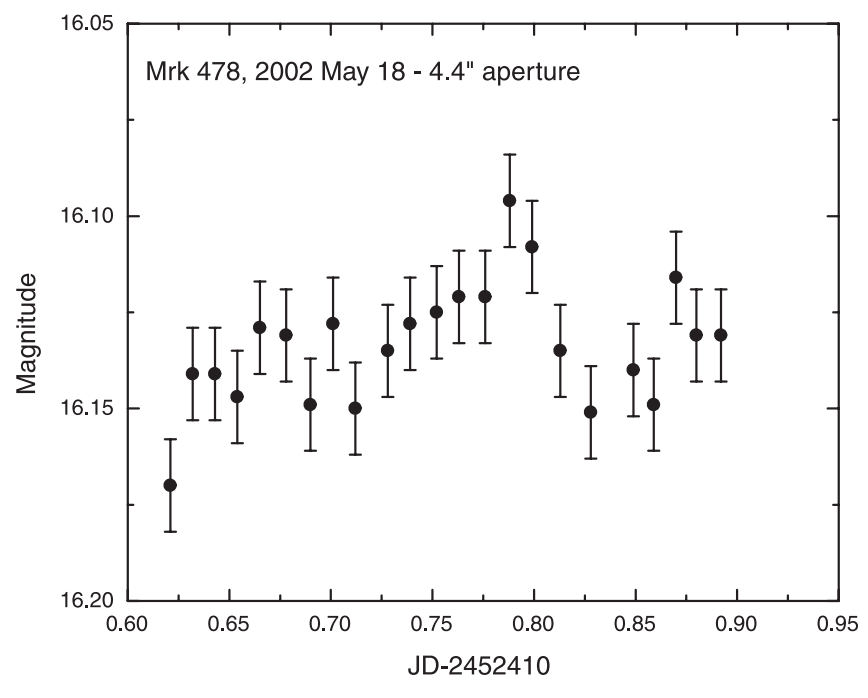

FIG. 9.-Apparent microvariability of Mrk 478 on 2002 May 18 when measured with a 4."4 aperture.

possible variations for two comparison stars of Ark 564 (see C. M. Gaskell et al. 2004, in preparation), but these are comparable to our measuring errors and have a negligible effect on our results, since for Ark 564 we are averaging comparisons with four stars.

\section{RESULTS}

\subsection{Subdiurnal Timescales}

All nights containing at least a dozen images of the same object, amounting to at least $3 \mathrm{hr}$ worth of continuous monitoring, were searched for evidence of microvariability. The significance was evaluated as described in $\S 4.2$. The results are shown in Table 8. For each test we give the probability of the possible variability arising by chance (i.e., the null hypothesis is no variability). As can be seen, the $F$-test reveals no correlated variation on the timescale of $\sim 30$ minutes. The Pearson correlation coefficient test and the Student $t$-test reveal trends with one-tailed significances of greater than $90 \%$

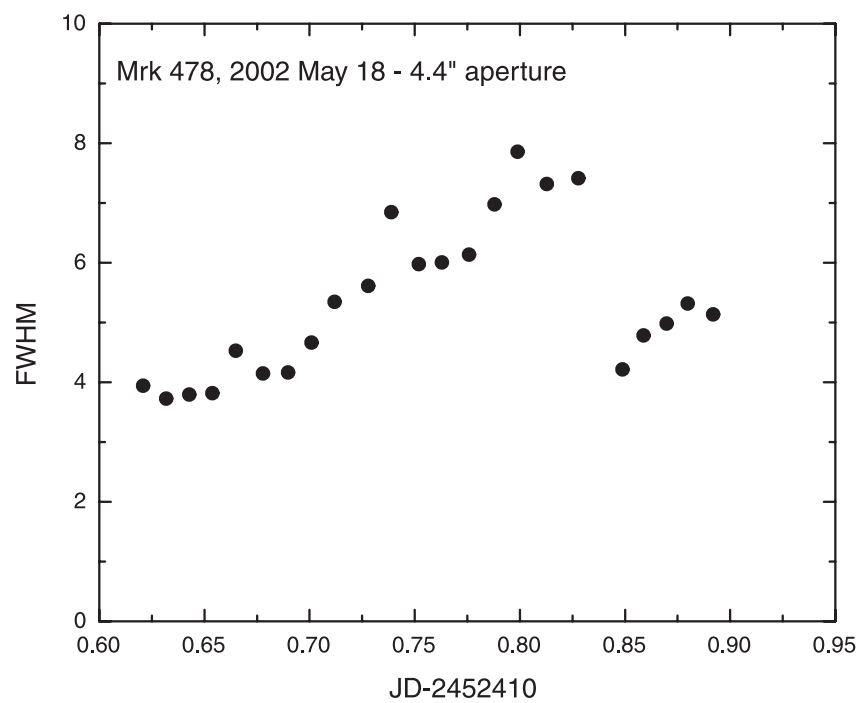

FIG. 10.-Variation of measured magnitude with image quality for Mrk 478 on 2002 May 18. 
TABLE 8

Results of Statistical Tests

\begin{tabular}{|c|c|c|c|c|c|}
\hline Name & Night & $t$-Test & Pearson & $F$-Test & Microvariability \\
\hline \multirow[t]{3}{*}{ 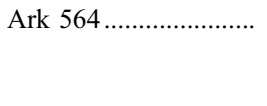 } & 1998 Oct 19 & 97.4 & 67.4 & 60.1 & \\
\hline & 1998 Nov 24 & 6.20 & 8.50 & 99.3 & $?$ \\
\hline & 2002 Oct 20 & 77.8 & 42.4 & 22.8 & \\
\hline \multirow{6}{*}{ Mrk 335 ....................... } & 2002 Oct 13 & 66.1 & 83.4 & 83.0 & \\
\hline & 2002 Oct 14 & 28.9 & 33.7 & 66.8 & \\
\hline & 2002 Oct 21 & 85.9 & 77.2 & 96.4 & \\
\hline & 2002 Oct 22 & 27.6 & 36.3 & 71.1 & \\
\hline & 2002 Nov 04 & 1.30 & 5.60 & 41.7 & $?$ \\
\hline & 2002 Nov 12 & 26.3 & 11.6 & 97.6 & \\
\hline \multirow[t]{4}{*}{ Mrk $359 \ldots \ldots \ldots \ldots \ldots \ldots \ldots$} & 2002 Nov 07 & 57.0 & 81.0 & 72.4 & \\
\hline & 2002 Nov 25 & 50.4 & 49.6 & 56.8 & \\
\hline & 2002 Dec 10 & 52.7 & 39.0 & 68.8 & \\
\hline & 2003 Jan 13 & 98.1 & 28.0 & 49.5 & \\
\hline \multirow[t]{12}{*}{ 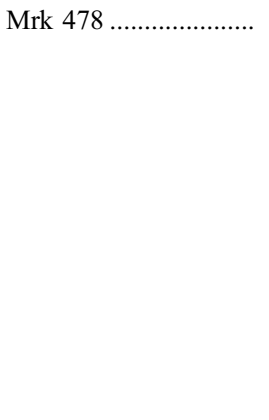 } & 2002 Mar 12 & 48.3 & 81.8 & 48.6 & \\
\hline & 2002 Apr 23 & 32.4 & 12.6 & 79.1 & \\
\hline & 2002 May 03 & 8.10 & 10.3 & 74.7 & \\
\hline & 2002 May 14 & 41.7 & 71.9 & 61.0 & \\
\hline & 2002 May 18 & 6.60 & 10.5 & 18.2 & \\
\hline & 2002 May 20 & 86.4 & 54.2 & 83.8 & \\
\hline & 2002 May 21 & 3.60 & 4.90 & 81.9 & $?$ \\
\hline & 2002 May 31 & 27.7 & 43.0 & 95.1 & \\
\hline & 2002 Jun 01 & 89.8 & 39.0 & 86.8 & \\
\hline & 2002 Jun 06 & 97.3 & 86.2 & 3.10 & \\
\hline & 2002 Jun 09 & 92.8 & 58.9 & 94.1 & \\
\hline & 2002 Jun 14 & 70.8 & 47.2 & 53.9 & \\
\hline \multirow[t]{3}{*}{ Mrk 493 ....................... } & 2002 Jun 27 & 8.30 & 7.30 & 77.2 & \\
\hline & 2002 Jun 28 & 47.8 & 6.60 & 82.1 & \\
\hline & 2003 Apr 09 & 37.6 & 11.0 & 96.9 & \\
\hline \multirow[t]{5}{*}{ 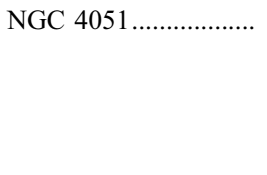 } & 2003 Feb 13 & 78.3 & 77.2 & 66.8 & \\
\hline & 2003 Feb 20 & 0.400 & 0.300 & 16.5 & Yes \\
\hline & 2003 Feb 21 & 39.6 & 5.90 & 26.2 & \\
\hline & 2003 Mar 06 & 62.2 & 71.9 & 68.8 & \\
\hline & 2003 Mar 14 & 16.7 & 9.90 & 73.9 & \\
\hline
\end{tabular}

on a timescale of several hours on five nights. However, because we are looking at 33 nights of monitoring, we would expect to get a significance of 1 in 10 about 3 times.

Allowing for the number of nights of observation, it is apparent from Table 8 that there is only one night with a high probability of microvariability: NGC 4051 on 2003 February 20 . There are four other nights on which the correlation and $t$-tests indicate possible variability at the $\sim 90 \%-95 \%$ significance level. Apparent variations on these nights were checked for spurious causes.

A 0.045 mag change was found in the light curve of NGC 4051 during the course of one night (see Fig. 11). Both the correlation and $t$-tests gave greater than $99.6 \%$ confidence that the variations were not due to chance. We checked for possible instrumental causes. Figure 11 shows the behavior of NGC 4051 on 2003 February 20 with respect to star 2, the brightest comparison star, and with respect to the average of the other three comparison stars. Both curves are in good agreement, attesting to the reliability of the comparison stars. The larger than average fluctuation in the third image is caused by a problem with star 2. Such problems are not unusual (see 3.1) and are consistent with our detector noise characteristics. Likewise, statistically there could also be a problem with the AGN in one or both of images 8 or 9 .

Figures 12 and 13 show plots of the $y$-position on the chip and the FWHM as a function of time. Although there are slight changes in the $y$-position during the night, comparable changes in the position on the chip are seen on other nights without affecting the magnitude. The two largest changes in the light curve (Fig. 11) and the $y$-position (Fig. 12) on the chip occur simultaneously. However, after this point the two plots behave differently. There were no peculiarities found either in the flat-fielding or in the dark subtraction. The

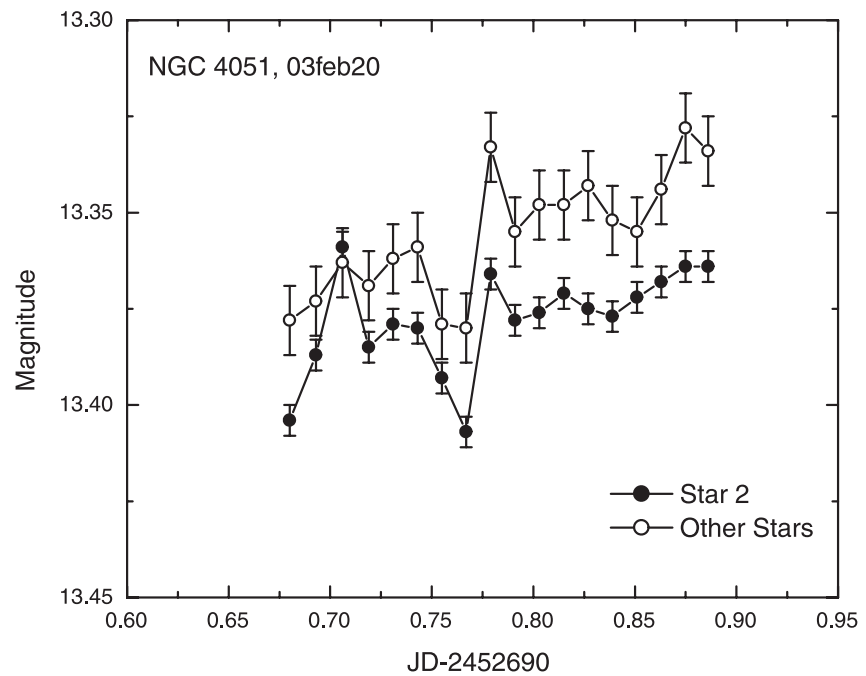

FIG. 11. $-V$-band light curve for NGC 4051 on 2003 February 20. The filled circles are the magnitudes measured relative just to star 2; the open circles are the magnitudes relative to the other comparison stars. 


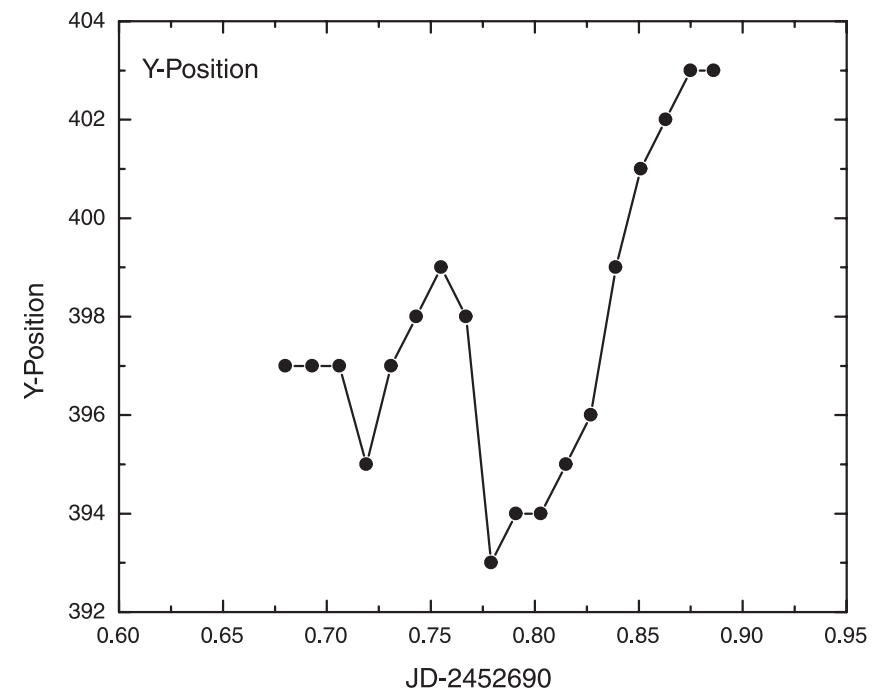

FIG. 12.-Variation of the $y$-position of the nucleus of NGC 4051 on the chip during the night of 2003 February 20.

$x$-position change (not shown) also shows no detailed correlation with the light curve. The image quality improved during the night, but the FWHM does not correlate in detail with the shape of the light curve. The $8^{\prime \prime}$ radius aperture magnitudes show no correlation with FWHM on any other night for NGC 4051 (or indeed for any other object). When the 4".4 radius aperture magnitudes do show a correlation with FWHM (see Figs. 8 and 10), it is in the opposite sense to that found here. We are thus unable to find artificial explanations of the apparent subdiurnal variations of NGC 4051 on 2003 February 20.

With the exception of the one night for NGC 4051, we therefore find very little evidence for variability on an intranight timescale. A study of microvariability in Seyfert galaxies (Carini et al. 2003) found only one Seyfert galaxy, the BLS1 Ark 120, in a sample of eight, which showed signs of subdiurnal activity. Their result for the NLS1 Mrk 335 was consistent with the null result obtained from our study. Jang \& Miller (1997) also found no evidence of microvariability in Mrk 335 in their

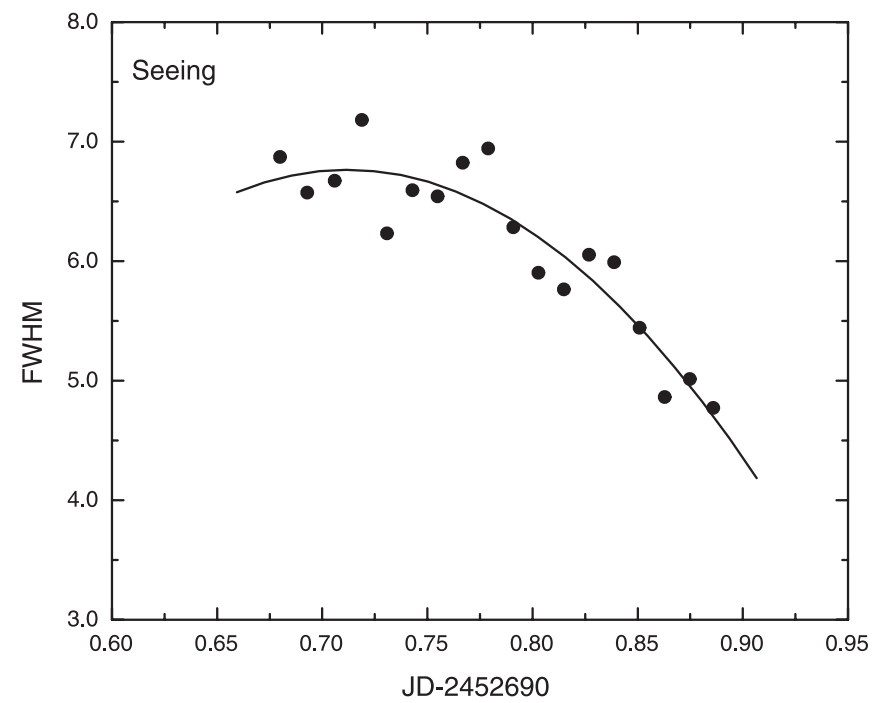

FIG. 13.-Variation of the image quality for images of NGC 4051 during the night of 2003 February 20.

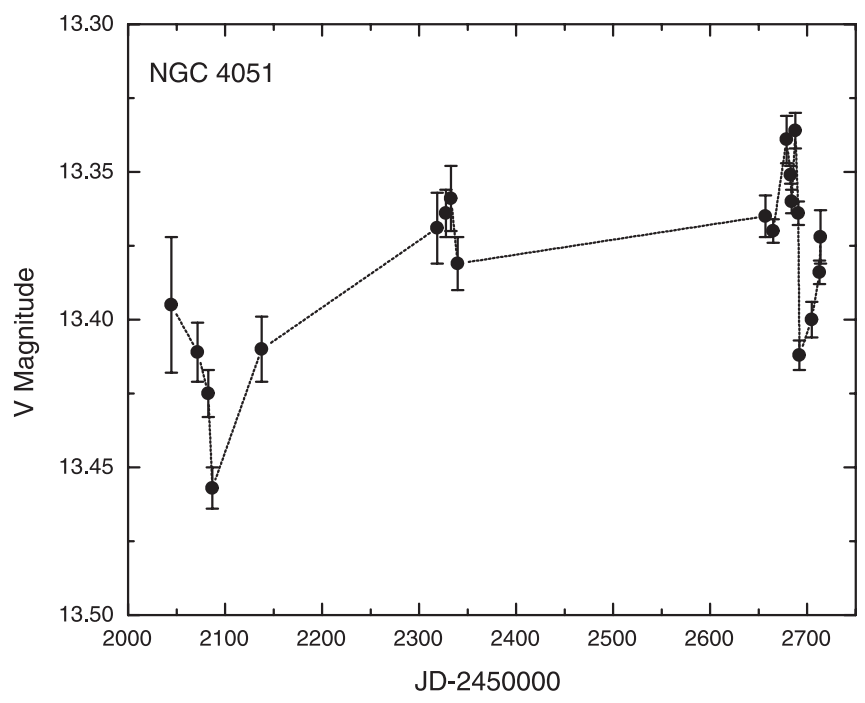

FIG. 14.-Long-term light curve for NGC 4051.

study of radio-quiet versus radio-loud quasars. Webb \& Malkan (2000a) searched for intranight variability in Seyfert 1 galaxies. No evidence for such variability was found, but they typically had only two images per night, compared with the more than 12 in this study, and their observational errors were about \pm 0.03 mag. Their sample included Mrk 478, an object for which we found no microvariations.

\subsection{Longer Timescales}

Long-term light curves from the nightly mean magnitudes given in Table 7 are plotted in Figures 14-18. As can be seen, variability is present for all six objects on a timescale of months to years.

In all photometric and spectroscopic studies of continuum variability there is contamination from the host galaxy. This varies with the size of the photometric or spectroscopic aperture used. In order to make a legitimate comparison, our magnitudes were scaled to small aperture sizes, which have been used in most spectrophotometric studies, such as those of the International AGN Watch. This was done by taking some

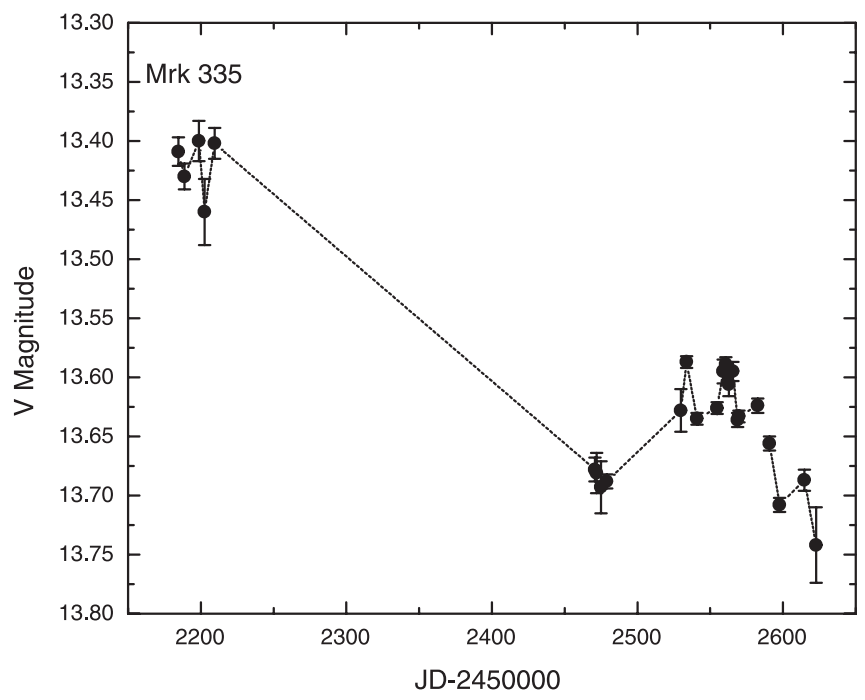

FIG. 15.-Long-term light curve for Mrk 335. 


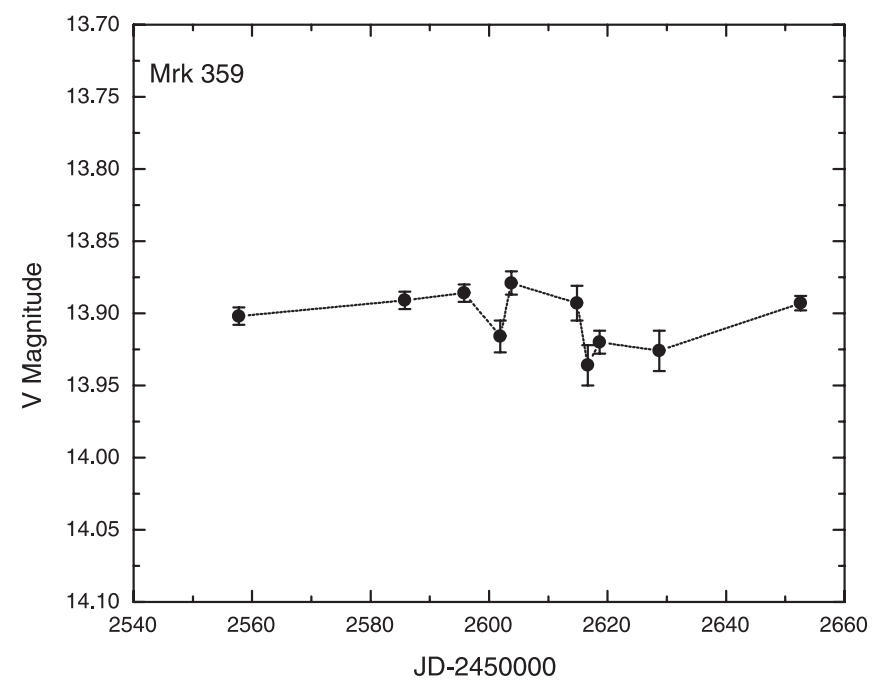

FIG. 16.-Long-term light curve for Mrk 359.

of our best images for each object (i.e., images having the lowest FWHM) and measuring the counts for successively smaller apertures. The ratio between the original aperture we used and the smallest practical aperture, 2".7, was found. This ratio was then used to scale our data by removing the extra host galaxy contribution in the larger aperture. This effectively gives a multiplicative correction factor for the amplitude of variability. These scale factors are given in Table 9. In Table 10 we give scaled seasonal standard deviations for our six NLS1's and also seasonal standard deviations for NGC 4051 from the International AGN Watch observations of Peterson et al. (2000). In Table 11 we give the seasonal standard deviations for eight non-NLS1 AGNs observed by the International AGN Watch (IAW) and others.

A comparison of the seasonal standard deviations of the NLS1's with the sample of BLS1's is given in Figure 19. From this we note the following:

1. For well-studied BLS1's (e.g., NGC 5548, NGC 4151, and $3 \mathrm{C} 273$ ), there is a range in standard deviation from season to season.

2. Some BLS1's appear on average to have higher seasonal standard deviations than others.

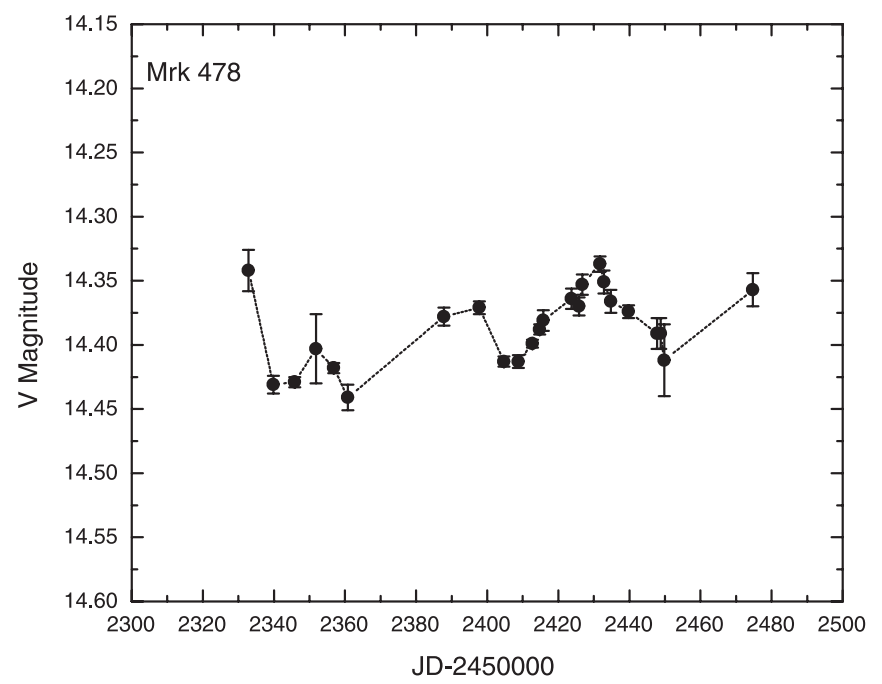

FIG. 17.- Long-term light curve for Mrk 478.

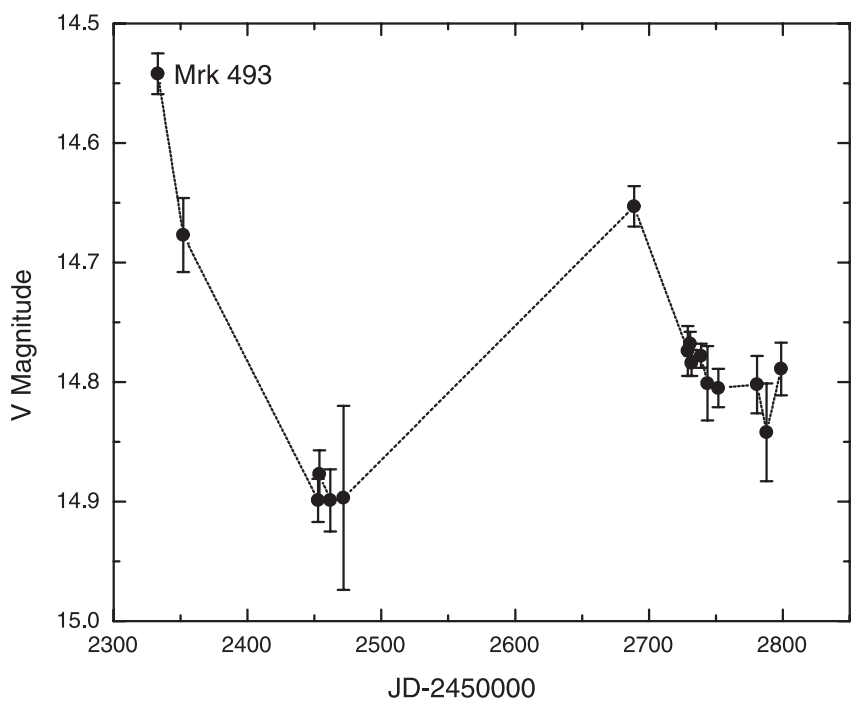

FIG. 18.- - Long-term light curve for Mrk 493.

3. NLS1's also show a spread in seasonal standard deviations.

4. The NLS1 seasonal standard deviations are in the lower half of the range of BLS1 standard deviations.

5. The well-studied NLS1 Ark 564 shows a range of seasonal standard deviations comparable to that of a BLS1 and in one season was as variable as the most variable BLS1 (see C. M. Gaskell et al. 2004, in preparation, for more detailed discussion).

\section{DISCUSSION}

\subsection{Short-Timescale Variability}

Our study clearly shows that high-amplitude variability in NLS1's is rare. As noted already, Miller et al. (2000) did, however, find an example of large-amplitude, rapid optical variability in IRAS 13224-3809. With this exception, NLS1's do not display rapid high-amplitude variability in the optical such as is seen in BL Lac objects or in the X-rays of NLS1's. Such short-timescale variability as there is must usually be of low amplitude. The study of Ferrara et al. (2001) also supports this conclusion. Interestingly, the one NLS1 in which we find reasonable convincing evidence for intranight variability is also the least luminous AGN in our sample. It is therefore the one with the least massive black hole (Peterson et al. 2000) and the one for which the most rapid variability would be expected. It is also perhaps interesting that this microvariability in NGC 4051 occurred when there was a rapid (1 day) drop in the flux (see Fig. 14).

Factors contributing to spurious artificial variations are numerous, and great care must be taken to account for them.

TABLE 9

Flux Ratio Scale Factors

\begin{tabular}{|c|c|}
\hline Object & Scale Factor \\
\hline Mrk $335 \ldots \ldots \ldots$ & 1.14 \\
\hline Mrk $359 \ldots \ldots \ldots$ & 1.93 \\
\hline 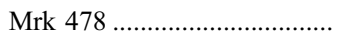 & 1.09 \\
\hline 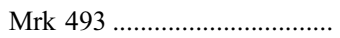 & 1.38 \\
\hline 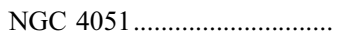 & 1.55 \\
\hline Ark $564 \ldots .$. & 1.24 \\
\hline
\end{tabular}


TABLE 10

The rms Seasonal Variability of NLS1's

\begin{tabular}{|c|c|c|}
\hline NLS1 & Variability & Average (Scaled) \\
\hline NGC $4051 \ldots \ldots$. & $0.036,0.045,0.048$ & 0.043 \\
\hline NGC $4051 \ldots \ldots$. & 0.014 & 0.022 \\
\hline Ark $564 \ldots \ldots \ldots \ldots$ & $0.025,0.054,0.147,0.045,0.014$ & 0.071 \\
\hline Mrk $335 \ldots \ldots \ldots$ & 0.023 & 0.026 \\
\hline Mrk $359 \ldots \ldots \ldots$ & 0.026 & 0.050 \\
\hline Mrk $478 \ldots \ldots \ldots$ & 0.025 & 0.027 \\
\hline Mrk $493 \ldots \ldots \ldots .$. & 0.050 & 0.069 \\
\hline
\end{tabular}

The inhomogeneity of data in previous variability studies is a problem. The errors must be well determined, and no sources of systematic error may be ignored. It is important to reduce errors because true variations could be hiding in the noise. Convincingly detecting microvariations is no small task.

Because of uncertainties in studies of non-NLS1 microvariability, it is not yet possible to make as detailed a comparison of the microvariability of NLS1's and BLS1's as we would like. As usual, more data are needed to further strengthen our findings. With all the factors that can lead to a false positive detection of microvariability, it is especially beneficial to have multiple observers to verify a detection of microvariability. This has generally not happened in the past.

\subsection{Long-Term Variability}

From Figure 19 it might seem that, on average, NLS1's are less variable than BLS1's, but before such a conclusion can be made, selection effects need to be understood. None of our objects were chosen on the basis of optical variability characteristics, but BLS1's are typically chosen for studies in the hope that they will show high-amplitude variations. This selection is based on prior variability history, and, as can be seen from Figure 19, some objects tend to be more variable than others. Thus, there is a selection effect if the non-NLS1 sample consists primarily of the most variable BLS1's, in which case the comparison between the variabilities of the two classes is not a valid one. Giannuzzo \& Stirpe (1996) and Giannuzzo et al. (1998) compared the Balmer line variability of NLS1's

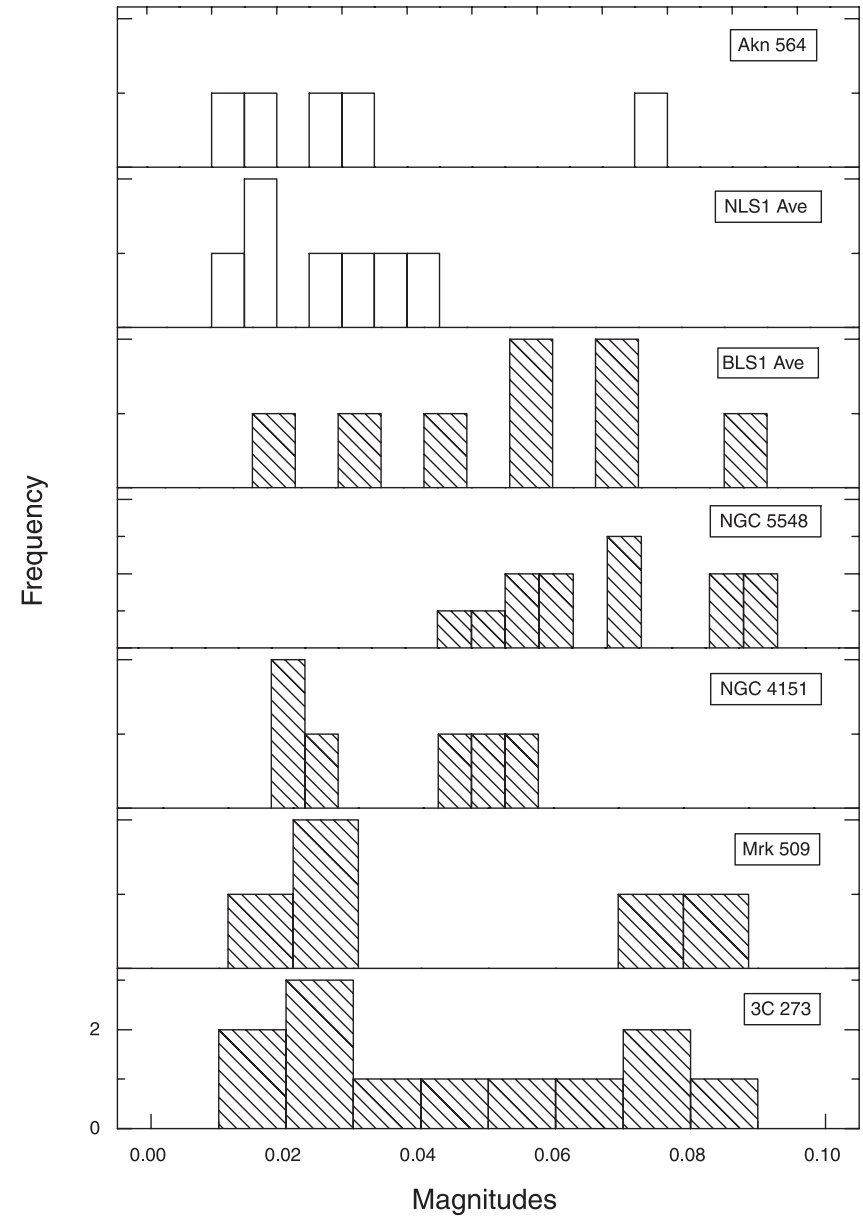

FIG. 19.-Distributions of rms $V$-band variability amplitudes for NLS1's (open squares) and BLS1's (hatched squares). For individual objects the seasonal variability is shown. For the NLS1 average and BLS1 average each square represents the mean of the variability over the seasons given in Tables 10 and 11 .

TABLE 11

The rms Seasonal Variability of BLS1's

\begin{tabular}{|c|c|c|}
\hline Source & rms Variability & Average \\
\hline NGC $3783^{\mathrm{a}, \mathrm{b}}$. & 0.085 & 0.085 \\
\hline NGC $4151^{\mathrm{a}, \mathrm{c}} \ldots \ldots \ldots \ldots . . .$. & $0.032,0.080,0.090,0.041,0.106,0.037$ & 0.064 \\
\hline NGC $5548^{\mathrm{a}, \mathrm{d}} \ldots \ldots \ldots \ldots \ldots$ & $0.137,0.136,0.099,0.172,0.100,0.112,0.085,0.161,0.113,0.107,0.165,0.173,0.130$ & 0.130 \\
\hline NGC $7469^{\mathrm{a}, \mathrm{e}} \ldots \ldots \ldots \ldots . . . .$. & 0.026 & 0.026 \\
\hline Mrk $279^{\mathrm{a}, \mathrm{f}} \ldots \ldots \ldots \ldots \ldots \ldots$ & $0.087,0.079$ & 0.083 \\
\hline Mrk $509^{\mathrm{a}, \mathrm{g}} \ldots \ldots \ldots \ldots \ldots \ldots . . . . . .$. & $0.132,0.076,0.142,0.087,0.086$ & 0.104 \\
\hline 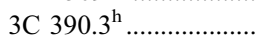 & $0.061,0.151,0.107$ & 0.106 \\
\hline $3 \mathrm{C} 273^{\mathrm{i}} \ldots \ldots \ldots \ldots \ldots \ldots \ldots$ & $0.030,0.016,0.059,0.073,0.026,0.082,0.040,0.067,0.014,0.023,0.032,0.077$ & 0.045 \\
\hline Average ....................... & $\ldots$ & 0.078 \\
\hline
\end{tabular}

a Observed by the IAW.

b Stirpe et al. 1994.

c Kaspi et al. 1996.

d Peterson et al. 1999.

e Collier et al. 1998.

f Santos-Lleó et al. 2001.

g Carone et al. 1996.

h Shapovalova et al. 2001.

i Türler et al. 1999. 
with that of NGC 5548, and Figure 19 shows that NGC 5548 is indeed more variable than the average BLS1.

We believe that, because of the lack of an adequate control sample of BLS1's, it is not yet possible to conclude that NLS1's are less variable in the optical than BLS1's. There is no evidence that the amplitude of variability of NLS1's and BLS1's is different.

\subsection{Implications}

That NLS1's vary in amplitude by so much so rapidly in the X-rays without any similar behavior in the lower energy end of the spectrum is a remarkable result and says something important about the structure of AGNs. The fact that NLS1's probably do not vary any differently than non-NLS1's in the visible continuum region is in itself another interesting finding.

These two findings rule out a situation in which the X-rays and the optical variations have a simple common origin. The source producing the X-ray variability cannot be the same as that producing the optical variations. If they both come from an accretion disk, for example, then they must be in distinctly different parts. If orientation plays a role in the NLS1 phenomenon, as it does through relativistic beaming, then only the X-ray emission is being enhanced.

Our results also do not support simple reprocessing, since the optical band should respond in some manner to the X-ray changes. That is, if the X-rays vary with a larger amplitude in NLS1's than in BLS1's, then the optical variations, if present, should also have a larger amplitude in NLS1's, even if the increase is small. We know that in general, NLS1's are highly variable in the X-rays, constantly varying rapidly (this is certainly true for one of our objects, Ark 564; Turner et al. 2001; Shemmer et al. 2001; Edelson et al. 2002), but we do not see any significant rapid optical variations in NLS1's (with the exception of one night for one object).

\section{CONCLUSIONS}

Our data show that, as a class, there is no evidence that NLS1's behave any differently than non-NLS1's in terms of variability. NLS1's can exhibit signs of variability on a timescale of hours, but such events are rare and of low amplitude. They do not exhibit the sort of remarkable variability seen in the X-rays. Longer term variability over a timescale of days to months is the norm, as it is with non-NLS1's.

Financial support for this work was provided in part by the University of Nebraska Layman Fund, the University of Nebraska Undergraduate Creative Activities and Research Experiences program, and by the National Science Foundation through grant AST 03-07912. We are grateful to Jeff Campbell, Kelly Crowley, Rene Goosmann, Beccie Grove, Rebecca Harbison, Lyle McConaughy, and Bradley W. Peterson for assistance in obtaining observations.
Boller, T., Brandt, W. N., Fabian, A. C., \& Fink, H. H. 1997, MNRAS, 289, 393

Boller, T., Brandt, W. N., \& Fink, H. H. 1996, A\&A, 305, 53

Boller, T., Trümper, J., Molendi, S., Fink, H., Schaeidt, S., Caulet, A., \& Dennefeld, M. 1993, A\&A, 279, 53

Boroson, T. A., \& Green, R. F. 1992, ApJS, 80, 109

Carini, M. T., Noble, J. C., \& Miller, H. R. 2003, AJ, 125, 1811

Carone, T. E., et al. 1996, ApJ, 471, 737

Cellone, S. A., Romero, G. E., \& Combi, J. A. 2000, AJ, 119, 1534

Collier, S. J., et al. 1998, ApJ, 500, 162

de Ruiter, H. R., \& Lub, J. 1986, A\&AS, 63, 59

Davidson, K., \& Kinman, T. D. 1978, ApJ, 225, 776

Edelson, R., Turner, T. J., Pounds, K., Vaughan, S., Markowitz, A., Marshall, H., Dobbie, P., \& Warwick, R. 2002, ApJ, 568, 610

Ferrara, E. C., Miller, H. R., McFarland, J. P., Williams, A. M., Wilson, J. W., Fried, R. E., \& Noble, J. C. 2001, in ASP Conf. Proc. 224, Probing the Physics of Active Galactic Nuclei, ed. B. M. Peterson, R. W. Pogge, \&

R. S. Polidan (San Francisco: ASP), 319

Gaskell, C. M. 1984, Astrophys. Lett., 24, 43 1985, ApJ, 291, 112

Gaskell, C. M., \& Klimek, E. S. 2003, Astron. Astrophys. Trans., 22, 661

Giannuzzo, M. E., Mignoli, M., Stirpe, G. M., \& Comastri, A. 1998, A\&A, 330,894

Giannuzzo, M. E., \& Stirpe, G. M. 1996, A\&A, 314, 419

Goodrich, R. W. 1989, ApJ, 342, 224

Howell, S. B. 1989, PASP, 101, 616

Jang, M., \& Miller, H. R. 1995, ApJ, 452, 582 1997, AJ, 114, 565

Kaspi, S., et al. 1996, ApJ, 470, 336

\section{REFERENCES}

Koski, A. T. 1978, ApJ, 223, 56

Merkulova, N. I. 2000, AJ, 119, 631

Miller, H. R., Ferrara, E. C., McFarland, J. P., Wilson, J. W., Daya, A. B., \& Fried, R. E. 2000, NewA Rev., 44, 539

Mushotzky, R. F., Done, C., \& Pounds, K. A. 1993, ARA\&A, 31, 717

Osterbrock, D. E., \& Pogge, R. W. 1985, ApJ, 297, 166

Penston, M. J., Penston, M. V., \& Sandage, A. R. 1971, PASP, 83, 783

Peterson, B. M., Pogge, R. W., Wanders, I., Smith, S. M., \& Romanishin, W. 1995, PASP, 107, 579

Peterson, B. M., et al. 1999, ApJ, 510, 659

2000, ApJ, 542, 161

Phillips, M. M. 1977, ApJ, 215, 746

Santos-Lleó, M., et al. 2001, A\&A, 369, 57

Sargent, W. L. W. 1968, ApJ, 152, L31

Shapovalova, A. I., et al. 2001, A\&A, 376, 775

Shemmer, O., et al. 2001, ApJ, 561, 162

Shrader, C. R., et al. 1990, BAAS, 22, 1193

Shuder, J. S., \& Osterbrock, D. E. 1981, ApJ, 250, 55

Stirpe, G. M., et al. 1994, ApJ, 425, 609

Türler, M., et al. 1999, A\&AS, 134, 89

Turner, T. J., Romano, P., George, I. M., Edelson, R., Collier, S. J., Mathur, S., \& Peterson, B. M. 2001, ApJ, 561, 131

Ulrich, M.-H., Maraschi, L., \& Urry, C. M. 1997, ARA\&A, 35, 445

Webb, W., \& Malkan, M. A. 2000a, ApJ, 540, 652 2000b, ApJS, 130, 165

Young, A. J., Crawford, C. S., Fabian, A. C., Brandt, W. N., \& O'Brien, P. T. 1999, MNRAS, 304, L46 
The Astrophysical Journal, 627:1072, 2005 July 10

(C) 2005. The American Astronomical Society. All rights reserved. Printed in U.S.A.

ERRATUM: “OPTICAL VARIABILITY OF NARROW-LINE SEYFERT 1 GALAXIES” (ApJ, 609, 69 [2004])

Elizabeth S. KlimeK, C. Martin Gaskell, and Cecelia H. Hedrick

Department of Physics and Astronomy, University of Nebraska

Due to various plotting errors, Figure 19 did not correctly represent the seasonal variability data presented in Tables 10 and 11 . Incorrect binning by the plotting software and inconsistent horizontal and vertical scaling did not make a comparison between the various histograms possible. The histogram representing Table 10 presented the unscaled instead of the scaled NLS1 variability averages and also mistakenly included an object in the $0.01-0.02$ bin that did not belong to the NLS1 sample.

Here we give a corrected version of Figure 19 with correct bin sizes and horizontal scaling. The erroneous data point has been removed, and the scaled NLS1 averages have been plotted. The errors in the original figure do not affect any conclusions made in this paper.

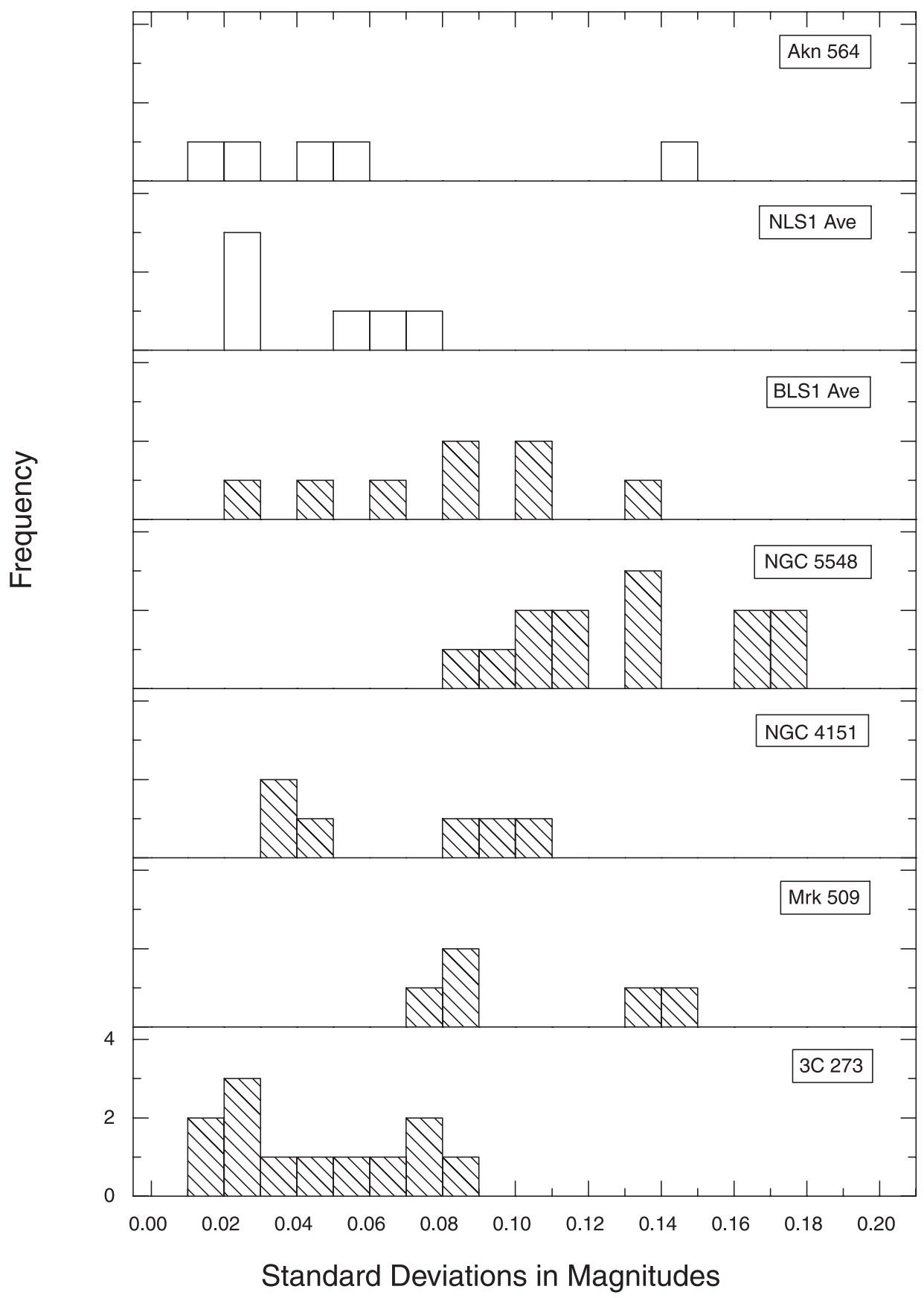

FIG. 19.-Distributions of rms V-band variability amplitudes for NLS1's (open squares) and BLS1's (hatched squares). For individual objects the seasonal variability is shown. For the NLS1 average and BLS1 average each square represents the mean of the variability over the seasons given in Tables 10 and 11. 